\title{
Estudio hidráulico bidimensional para el proyecto de rehabilitación del meandro "Los Garridos" en el río Jarama (Algete, Madrid)
}

Two-dimensional hydraulic study for the rehabilitation project of the meander "Los Garridos" in Jarama river (Algete, Madrid)

Larrañaga, A.; Carrero, L. ${ }^{1}$; Fernández, J.A. ${ }^{1}$

${ }^{1}$ Grupo de Investigación Ecología y Gestión Forestal Sostenible. ECOGESFOR-UPM. E.T.S.I. Montes, Forestal y del Medio Natural. Avda. Ramiro de Maeztu, s/n. Ciudad Universitaria. 28040 Madrid. 


\section{Resumen}

El meandro de "Los Garridos", en el río Jarama, es un claro ejemplo del resultado de la alteración del régimen hidrológico y de profundas modificaciones geomorfológicas. La fotografía aérea de 1975 muestra cómo el inicio de una explotación de áridos en el entorno del meandro origina una corta artificial que se ha mantenido como único cauce y que ha hecho que el meandro no se haya activado desde entonces, presentando actualmente signos de profundo decaimiento de lo que en su día fue un espacio fluvial dinámico y complejo.

Para evaluar escenarios de actuación que permitan rehabilitar hidráulica y ambientalmente este meandro se ha puesto a punto un modelo hidráulico bidimensional (Iber). La caracterización del comportamiento hidráulico obtenido para cada escenario, ha permitido aplicar indicadores ambientales y de coste para poder seleccionar el más eficiente de cara a utilizarlo para el proyecto de rehabilitación.

Palabras clave: alteración hidromorfológica, corta de meandro, espacio fluvial, modelización hidráulica, restauración de ríos.

\section{Summary}

The "Los Garridos" meander in the Jarama river is an example of the consequences of the alteration of the hydrological regime and geomorphological modifications. The aerial photograph from 1975 shows how the human activities in the area around the meander caused an artificial cutoff, which has been maintained as the only channel ever since. As a result of these changes, the meander is not active anymore and, currently showing signs of strong decay, a meander that was once a dynamic and complex fluvial space.

A two-dimensional hydraulic model (Iber) has been developed in order to provide alternative scenarios for the hydraulic and environmental restoration of the meander. To evaluate the efficiency of each scenario, four environmental and cost indicators were chosen, in order to select the most efficient one to be used for the rehabilitation project.

Keywords: channel dynamic, hydromorphological alteration, hydraulic model, meander cutoff, river restoration. 


\section{Introducción}

La dinámica morfológica de los ríos es el resultado del balance entre la energía potencial que el relieve define, el transporte de caudales líquidos y sólidos y la disipación resultante de la interacción de estos flujos con el lecho y orillas. Esa dinámica morfológica es imprescindible para mantener la integridad de componentes, procesos y funciones del ecosistema fluvial, un ecosistema que, vinculado a su morfología es, como ésta, dinámico.

El río, su forma, tanto en planta como en sección, está en movimiento permanente. Un movimiento que, en general, se manifiesta en equilibrio dinámico con unas formas básicas, en las que la meandriforme es, junto con el trenzado, una de las más habituales.

El espacio creado como consecuencia de los procesos de depósito en la parte interior de las curvas de los meandros ha sido muy atractivo para la agricultura, para explotaciones forestales y también para extracción de áridos. Esta última actividad ha sido especialmente intensa durante el último cuarto del pasado siglo, especialmente en cauces próximos a grandes ciudades.

En ocasiones, para garantizar que esos trabajos de extracción no se viesen interrumpidos por crecidas, se hicieron cortas y modificaciones del cauce que alteraron definitivamente la dinámica del meandro, sin posibilidades de auto-recuperación. Es el caso del meandro "Los Garridos", en el tramo medio del río Jarama: debido a las alteraciones morfológicas sufridas en su entorno para optimizar la actividad extractiva de áridos, acentuadas por la regulación de caudales debida a los numerosos embalses construidos aguas arriba, el meandro se ha desconectado del río. La pérdida de este elemento implica una reducción de complejidad y diversidad morfológica, y con ella del espacio ripario asociado, así como del biotopo hidráulico, especialmente diverso y dinámico en estas formas fluviales.

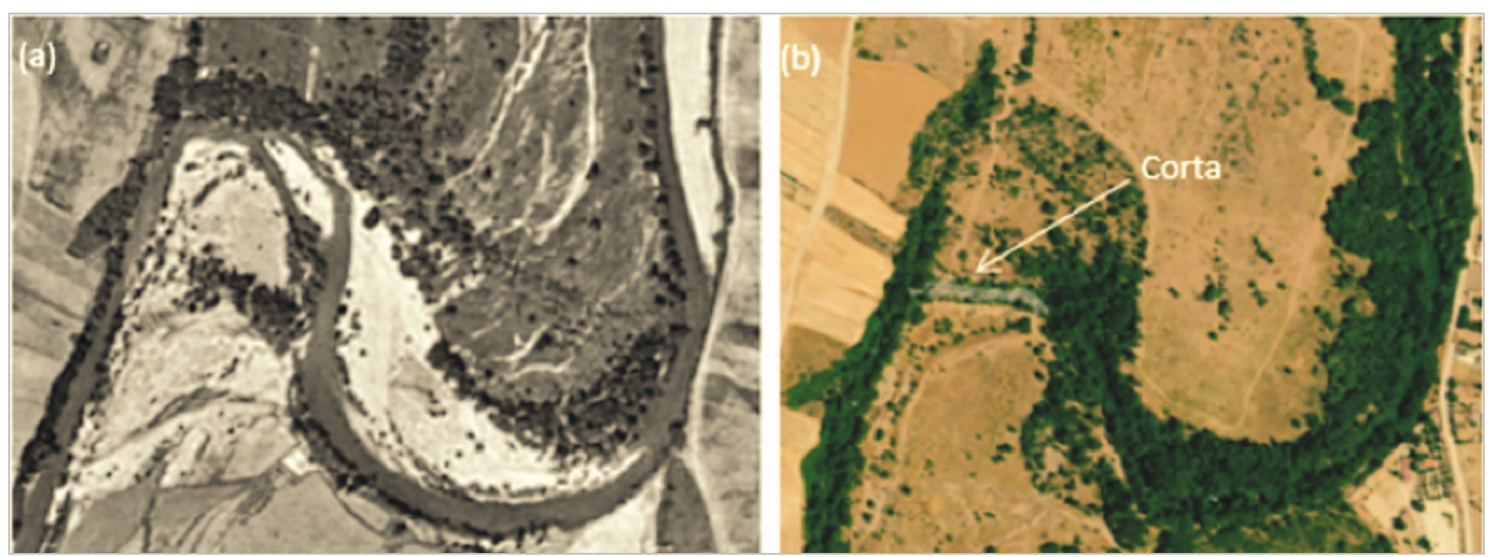

Figura 1. Evolución de la morfología del meandro Los Garridos. (a) Cauce y espacio fluvial activo asociado a la dinámica del meandro en 1954. (b) Estado actual (foto aérea de 2019):se puede apreciar la corta que se hizo para facilitar la explotación de áridos y la pérdida, casi desaparición, de vegetación riparia en buena parte de lo que fue la traza del meandro. 
La Figura 1 pone en evidencia la pérdida de la dinámica morfológica del meandro, hasta su práctica desaparición. ¿Es posible recuperar hidráulica y funcionalmente el meandro? Para poder contestar a esta pregunta es necesario, como punto de partida:

1. Caracterizar el comportamiento hidráulico actual del tramo.

2. Establecer escenarios de actuación para la recuperación de su funcionalidad.

3. Desarrollar simulaciones hidráulicas para caracterizar la respuesta del río a cada uno de los escenarios considerados.

4. Evaluar los resultados de las simulaciones aplicando indicadores ambientales y de coste.

El escenario así seleccionado podrá utilizarse para desarrollar el oportuno proyecto de rehabilitación hidráulica y ambiental del meandro.

\section{Material y Métodos}

\subsection{Zona de estudio}

La zona de estudio (Figura 2) se encuentra en el tramo medio del río Jarama, uno de los principales afluentes del Tajo, concretamente entre los municipios de Algete y Fuente del Saz de Jarama (Madrid). La principal alteración hidromorfológica del meandro ha sido la corta que, desde mediados de los años 70 del pasado siglo, lo desco-

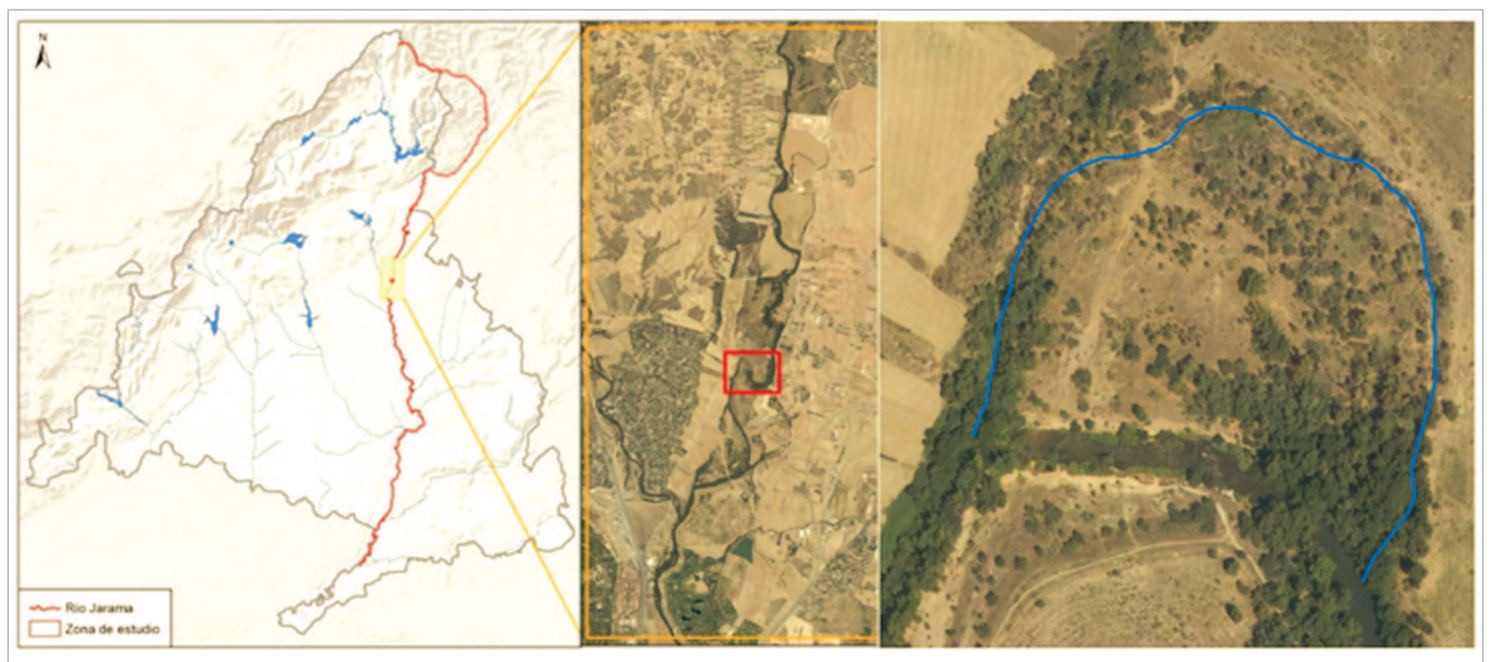

Figura 2. Localización de la zona de estudio. La línea azul representa el thalweg del meandro abandonado. 
nectó de su dinámica fluvial. Esa corta, con apenas 200 metros, desvinculó del cauce principal más de 560 metros de meandro. Actualmente el tramo más distal del meandro ha perdido su vegetación de ribera, lo que pone en evidencia la pérdida ambiental de este elemento morfológico como parte activa del ecosistema fluvial.

\subsection{Secuencia de trabajo}

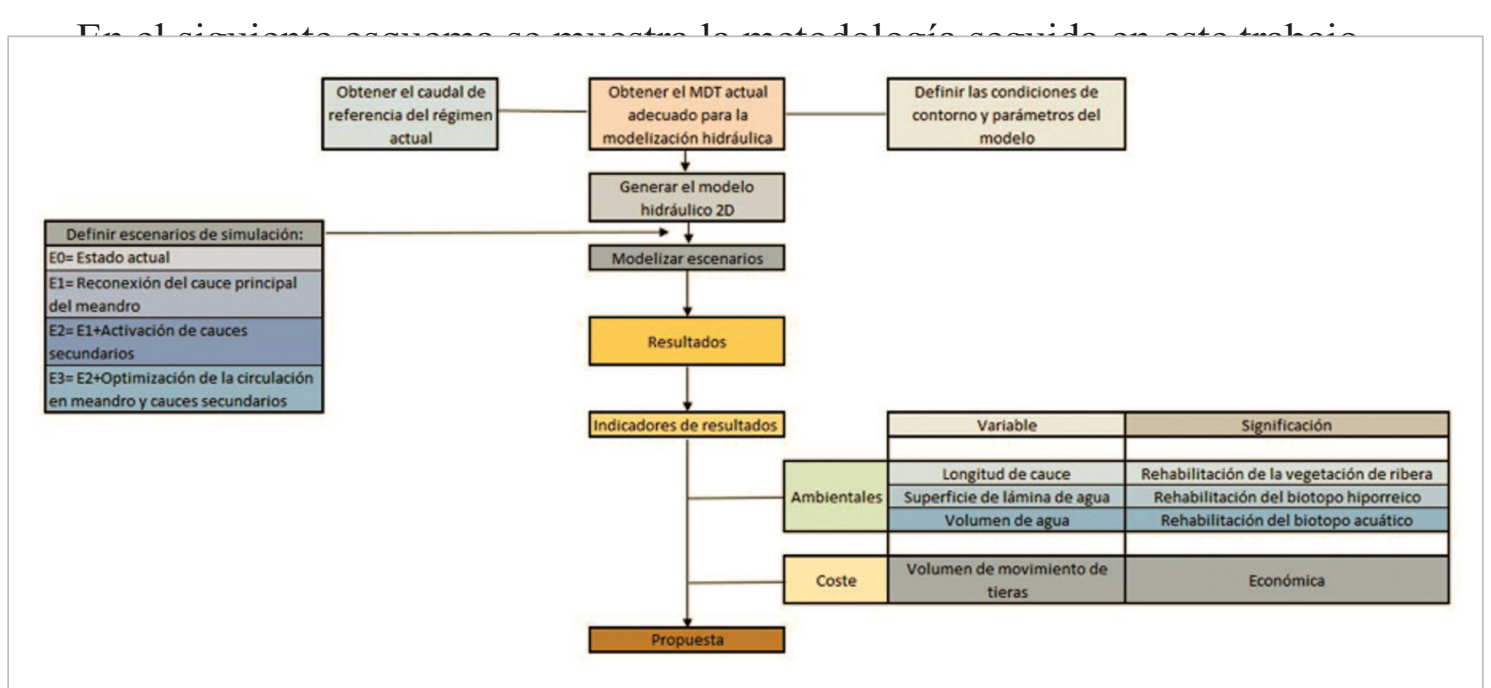

Figura 3. Diagrama del proceso seguido en el trabajo.

\subsubsection{Obtención del caudal de referencia}

Para conocer el caudal de referencia del régimen que actualmente presenta el cauce, se ha consultado los registros que ofrece el anuario de aforos del CEDEX de las estaciones 3051 y 3054 para el periodo 1980-2016. A partir de esos datos se construyó la curva de caudales clasificados (Vogel y Fennessey, 1995) y se determinaron los caudales correspondientes a los percentiles de excedencia 5, 20 y 50 que se usaron como condición de contorno a la entrada en el modelo hidráulico (Tabla 1) (Figura 4).

Tabla 1. Valores de caudales medios diarios clasificados escogidos para la simulación hidráulica.

$\begin{array}{lccc}\text { Excedencia (\%) } & 5 & 20 & 50 \\ \text { Caudal }\left(\mathbf{m}^{3} \mathbf{s}^{-1}\right) & 20,9 & 4,6 & 1,7\end{array}$




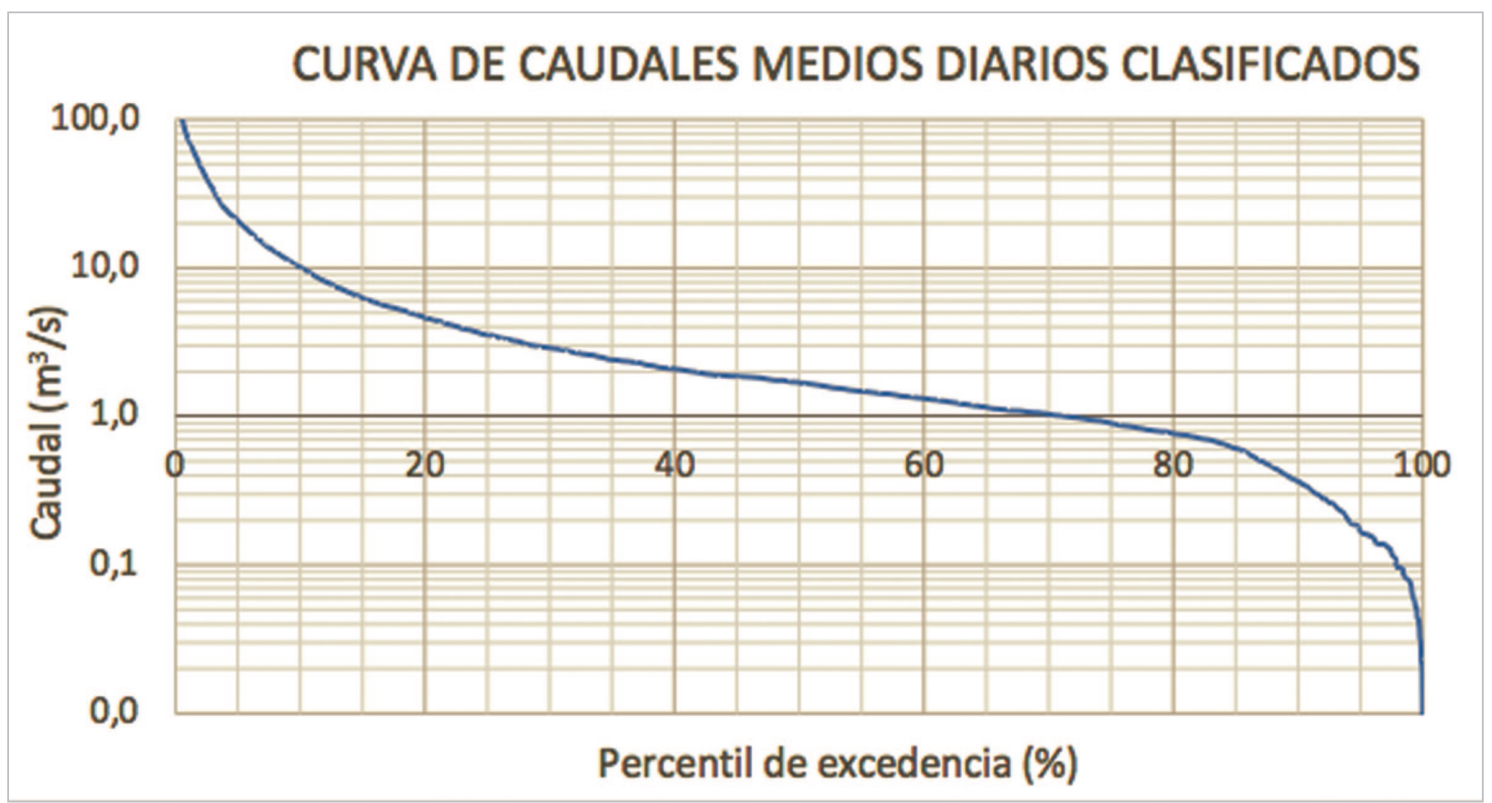

Figura 4. Curva de caudales medios diarios clasificados.

Fuente: Elaboración propia a partir de los datos del Anuario de Aforos (CEDEX).

\subsubsection{Obtención del Modelo Digital del Terreno}

A partir de la nube de puntos $3 \mathrm{D}$ correspondiente a la $2^{\mathrm{a}}$ cobertura LiDAR (densidad de 1 punto $\mathrm{m}^{-2}$ y precisión de $20 \mathrm{~cm}$ RMSE Z) que suministra el Centro Nacional de Información Geográfica (CNIG) y siguiendo como referencia la Guía Metodológica para el Desarrollo del Sistema Nacional de Cartografía de Zonas Inundables (2012), se obtuvo el modelo digital del terreno con tamaño de celda 1m (MDT), que sirvió de base topográfica para las simulaciones hidráulicas.

\subsubsection{Modelo 2D}

Para caracterizar el comportamiento hidráulico se ha utilizado el programa Iber (Bladé et al., 2014). Iber es un software libre (www.iberaula.es) para la modelización bidimensional de flujo de agua en lámina libre con aplicaciones a la hidráulica y morfología fluvial. La utilización de un modelo bidimensional en este estudio fue necesaria por:

1. La posible entrada de agua al meandro por varias vías, con orientaciones del flujo muy distintas a las que se presentan en el cauce principal.

2. La evidencia de brazos que, cuando el meandro era funcional, desbordaban desde el antiguo cauce sobre la parte interior de la curva del meandro, inundándola.

3. La necesidad de simular el comportamiento del flujo una vez rehabilitada la co- 
nexión hidráulica con el meandro hoy abandonado, flujo que presentará un campo de velocidades difícilmente compatible con la hipótesis unidimensional de los modelos 1D.

\subsubsection{Condición de contorno a la salida}

Los modelos hidráulicos necesitan fijar una condición a la salida del tramo para realizar la simulación. Esta condición se puede conocer con precisión siempre que se monitorice la sección. Dado que esta circunstancia no se daba, se optó por validarla realizando un análisis de sensibilidad. Se consideró como condición de salida las distintas opciones que presenta el programa -vertedero rectangular en lámina libre y nivel de lámina de agua dado-, y se planteó un análisis de sensibilidad sobre las variables calado y velocidad, modificando la condición de salida de referencia (CSR) en \pm 0.5 metros. Se asume como CSR un vertedero rectangular en lámina libre, con coeficiente de gasto 1.6 y cota de vertedero coincidente con el lecho y se realizó el análisis para la condición de entrada igual al caudal con percentil de excedencia del 20\% (Q20\%) y del 50\% (Q50\%). Se consideró que no había diferencias significativas cuando la diferencia de variables (valores de referencia respecto a valores obtenidos) no superaban los umbrales establecidos: $0.02 \mathrm{~m}$ para calados y $0.04 \mathrm{~m} \mathrm{~s}^{-1}$ para velocidades. Posteriormente se analizó el cumplimiento de estos umbrales calculando el porcentaje de superficie simulada que se encontraba bajo el umbral.

Los resultados mostraron que, tanto para el calado como para la velocidad, prácticamente la totalidad de la superficie de estudio se encontraba por debajo de los umbrales establecidos ( $85 \%$ de la superficie para el calado y $70 \%$ para velocidad) (Tabla 2 ). Por lo tanto, las diferencias en las estimas de calado y velocidad fueron despreciables. Este resultado permite trabajar con la CRS de vertedero rectangular con garantía de su adecuación.

Tabla 2. Resultados de \% de superficie con diferencia de calado y velocidad inferior al umbral cuando se compara la condición de salida de referencia (CSR) y la condición de cota de lámina de agua media (CLA).

\begin{tabular}{ccc}
\hline & \multicolumn{3}{c}{ \% Superficie } \\
\hline Diferencia calado $(<\mathbf{0 . 0 2} \mathbf{~ m})$ & $\mathbf{Q 2 0 \%}$ & $\mathbf{Q 5 0 \%}$ \\
CSR vs. CLA+0.5 & 86.8 & 88.6 \\
CSR vs. CLA-0.5 & 99.3 & 99.9 \\
& & \\
Diferencia velocidad $(<\mathbf{0 . 0 4}$ m s-1) & 89.5 & 90.2 \\
CSR vs. CLA+0.5 & 98.7 & 98.8 \\
CSR vs. CLA-0.5 & & \\
\hline
\end{tabular}




\subsubsection{Parámetros del modelo}

Normalmente, cuando se realizan modelizaciones hidráulicas en $2 \mathrm{D}$, el parámetro de rugosidad se ajusta mediante una calibración del modelo con datos de campo. Al no disponer de datos para abordar esa calibración, se asignaron los valores de la n de Manning tomando como referencia el anejo V de la Guía Metodológica para la Cartografía de Zonas Inundables y se validaron con un análisis de sensibilidad. Para cada tipo de rugosidad se tomó el valor mínimo y máximo del intervalo propuesto en la bibliografía (Tabla 3) y se estudió su influencia tomando los mismos umbrales y criterios que en el análisis para la condición de salida.

Tabla 3. Rangos de n de Manning para diferentes usos del suelo.

\begin{tabular}{cc} 
Uso del suelo & n de Manning \\
Erial & $0,03-0,035$ \\
Arbolado denso & $0,07-0,1$ \\
Arbolado disperso & $0,05-0,06$ \\
Roca & $0,035-0,04$ \\
Arenas & $0,03-0,035$ \\
\hline
\end{tabular}

Fuente: Guía Metodológica para el Desarrollo del Sistema Nacional de Cartografía de Zonas Inundables

Los resultados mostraron que las diferencias cumplían, para ambas variables, los umbrales establecidos. Estos resultados permitieron tomar la decisión de escoger el valor promedio de cada rango de valores de $\mathrm{n}$ de Manning como valor de rugosidad para las simulaciones.

Otro parámetro necesario para la simulación es la malla de cálculo. En el estudio se usó una malla no estructurada de elementos finitos llegando a la máxima resolución que permitía el MDT. La malla generada está compuesta por 109191 elementos y 55260 nodos.

\subsubsection{Escenarios de simulación}

Se modelizaron cuatro escenarios, siendo el escenario 0 la situación actual y los escenarios 1, 2 y 3 las alternativas planteadas para reconectar el antiguo meandro.

Para el escenario 0 se modelizó las condiciones topográficas actuales y se identificaron los obstáculos topográficos que dificultaban la circulación del flujo y la cota de lámina de agua que se alcanzaba cuando circulaban los distintos caudales.

En el escenario 1 se planteó una excavación a la cota identificada en el escenario 0 en el tapón que se localiza entre el cauce actual y el cauce principal abandonado del antiguo meandro (Figura 5). 


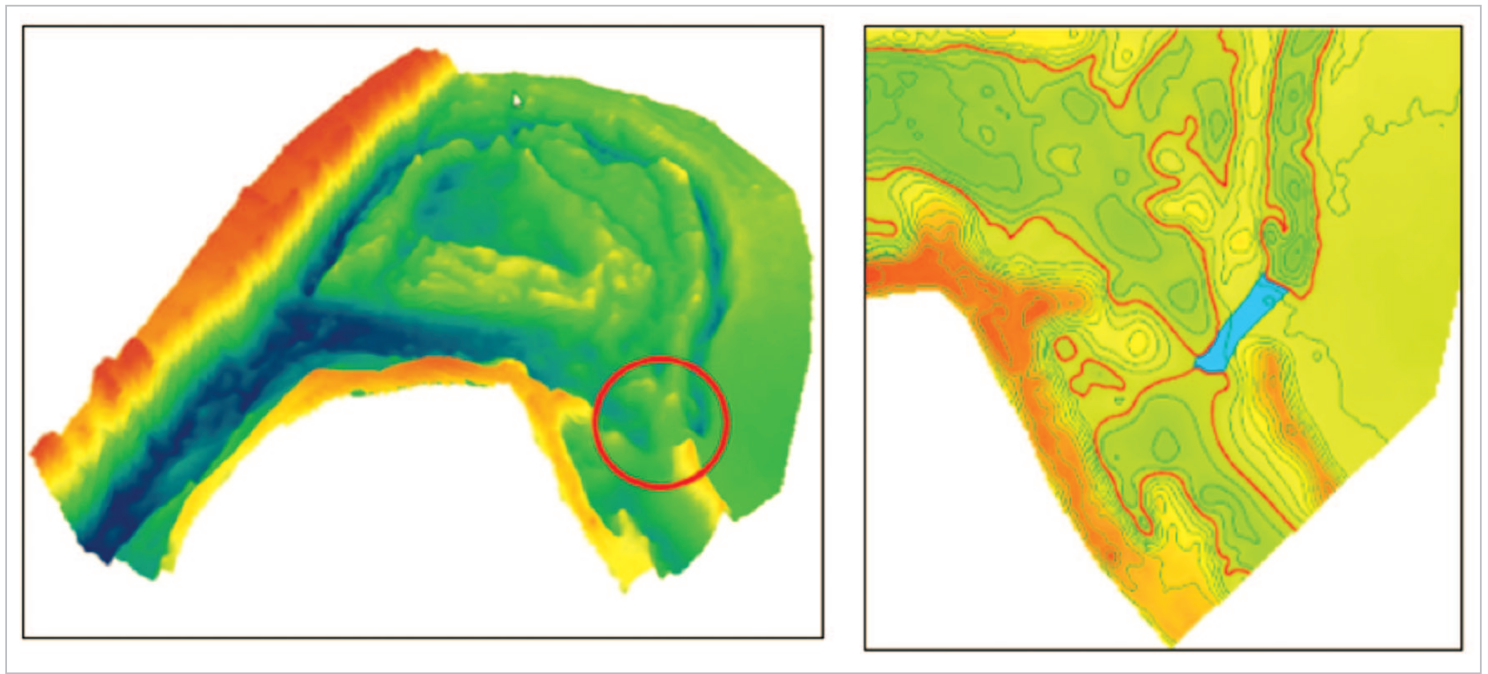

Figura 5. Localización del tapón en la entrada del meandro (izquierda) y propuesta de modificación a cota definida (línea roja) sobre MDT para el escenario 0 (derecha).

Para el escenario 2 se localizaron cauces secundarios en el interior del meandro que, con movimientos de tierra relativamente pequeños, podrían activarse. En la $\mathrm{Fi}$ gura 6 se muestran dichas zonas (zona M, CS1 y CS2).

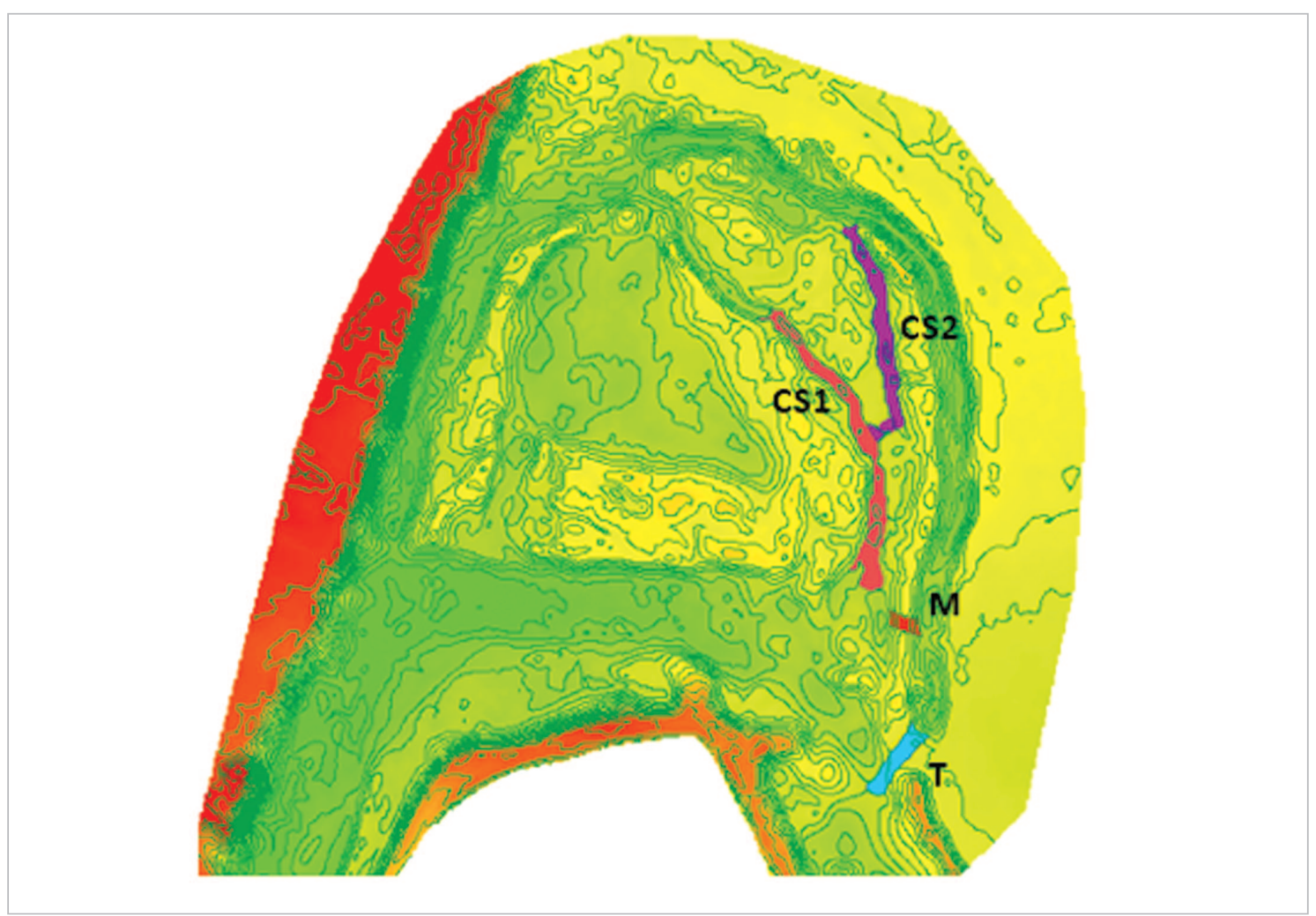

Figura 6. Propuesta de modificaciones topográficas sobre el MDT para el escenario 2 
El objetivo del escenario 3 era que los cauces secundarios se activaran con más frecuencia y que se garantizase la continuidad del flujo en el cauce principal del antiguo meandro. Para ello se propuso:

1. Rebajar la cota de la excavación del tapón de entrada y ensancharla (T1).

2. Ensanchar los cauces en las zonas M2 y M3.

3. Rebajar la cota en los cauces secundarios CS1 y CS2 (Figura 7).

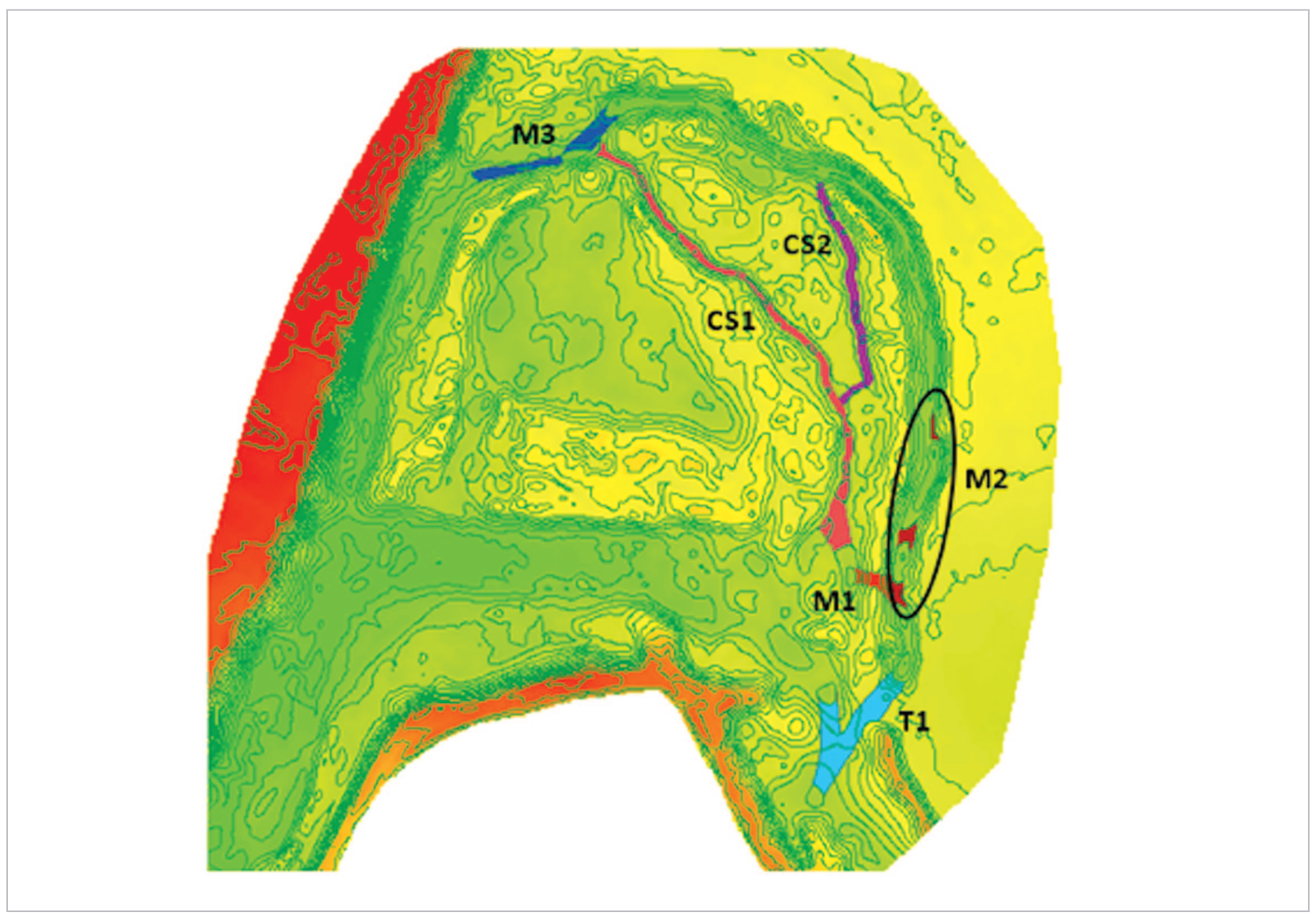

Figura 7. Propuesta de modificación sobre el MDT para el escenario 3

Se han realizado un total de 23 simulaciones: 6 para el análisis de sensibilidad de la condición de salida, 4 para el análisis de sensibilidad de la rugosidad, 4 para la caracterización hidráulica definitiva y 9 para las simular las distintas alternativas de reconexión del meandro. 


\section{Resultados y discusión}

\subsection{Escenario 0. Estado actual.}

En la Figura 8 se muestra la situación actual del meandro, sin realizar ninguna intervención. En ella se representan los calados obtenidos para los caudales Q50\%, Q20\% y Q5\%.

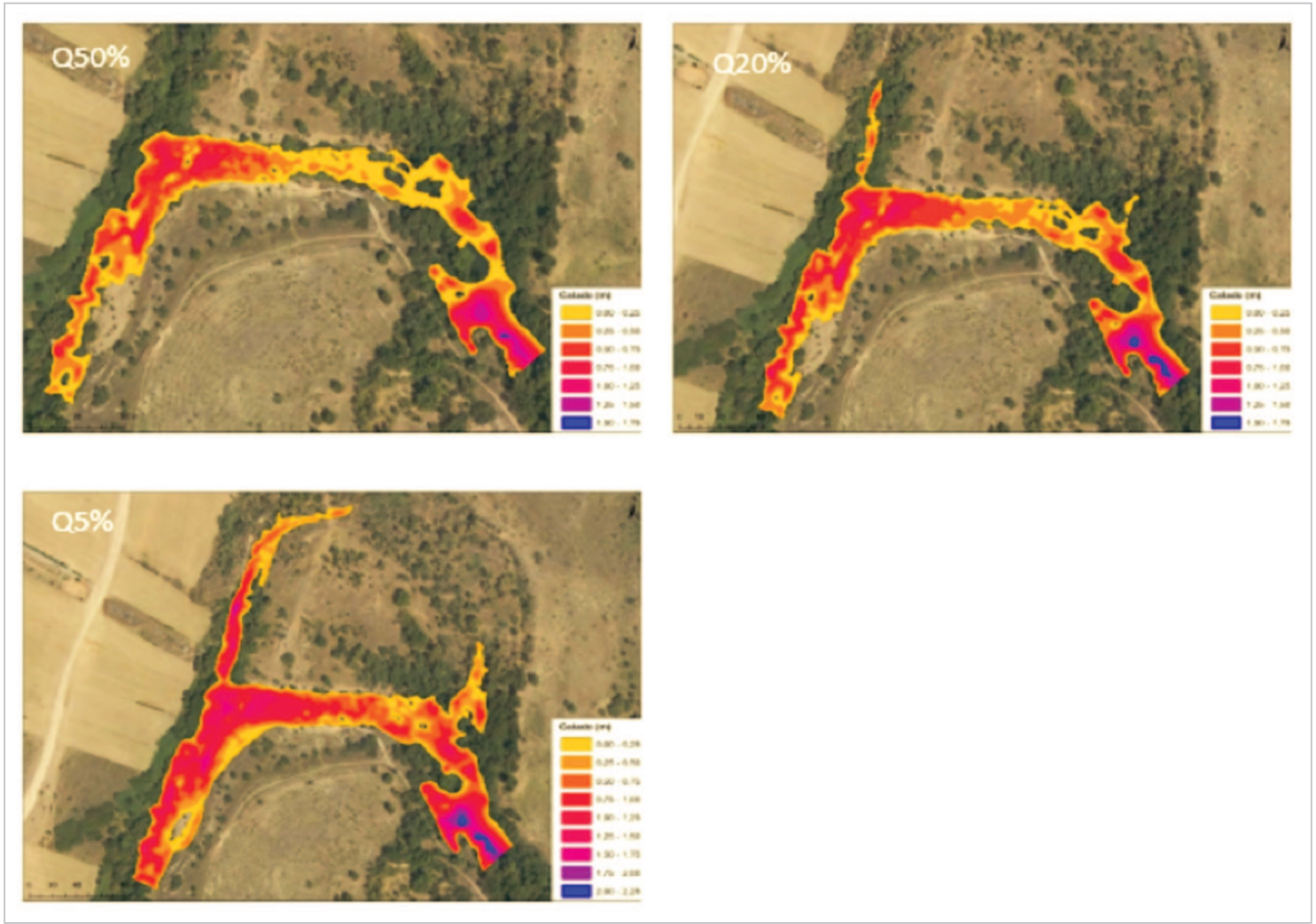

Figura 8. Resultado de calados alcanzados cuando circulan los caudales Q50\%, Q20\% y Q5\% en el escenario 0 .

Los resultados muestran que actualmente la funcionalidad hidráulica del meandro está condicionada por el tapón que se encuentra en su entrada y que hace que el agua circule por la corta. El antiguo cauce no es funcional ni tan siquiera para Q5\%, y para Q20\% el agua no entra al meandro por la que fue la sección de entrada, sino que presenta un remonte en lo que fue el tramo de salida. Por lo tanto, desde el punto de vista tanto hidráulico como ambiental, puede afirmarse que, para el régimen de caudales actual, el meandro no es funcional.

\subsection{Escenario 1. Reconexión del cauce principal del meandro.}

Los resultados arrojan que con el escenario 1 se conseguiría reconectar el meandro para el caudal Q50\%, lo que supone que el antiguo meandro estaría activo, 
como promedio, la mitad del año. Sin embargo, ninguno de los cauces secundarios que presentaba cuando era funcional se activan, ni tan siquiera para Q5\% (Figura 9).
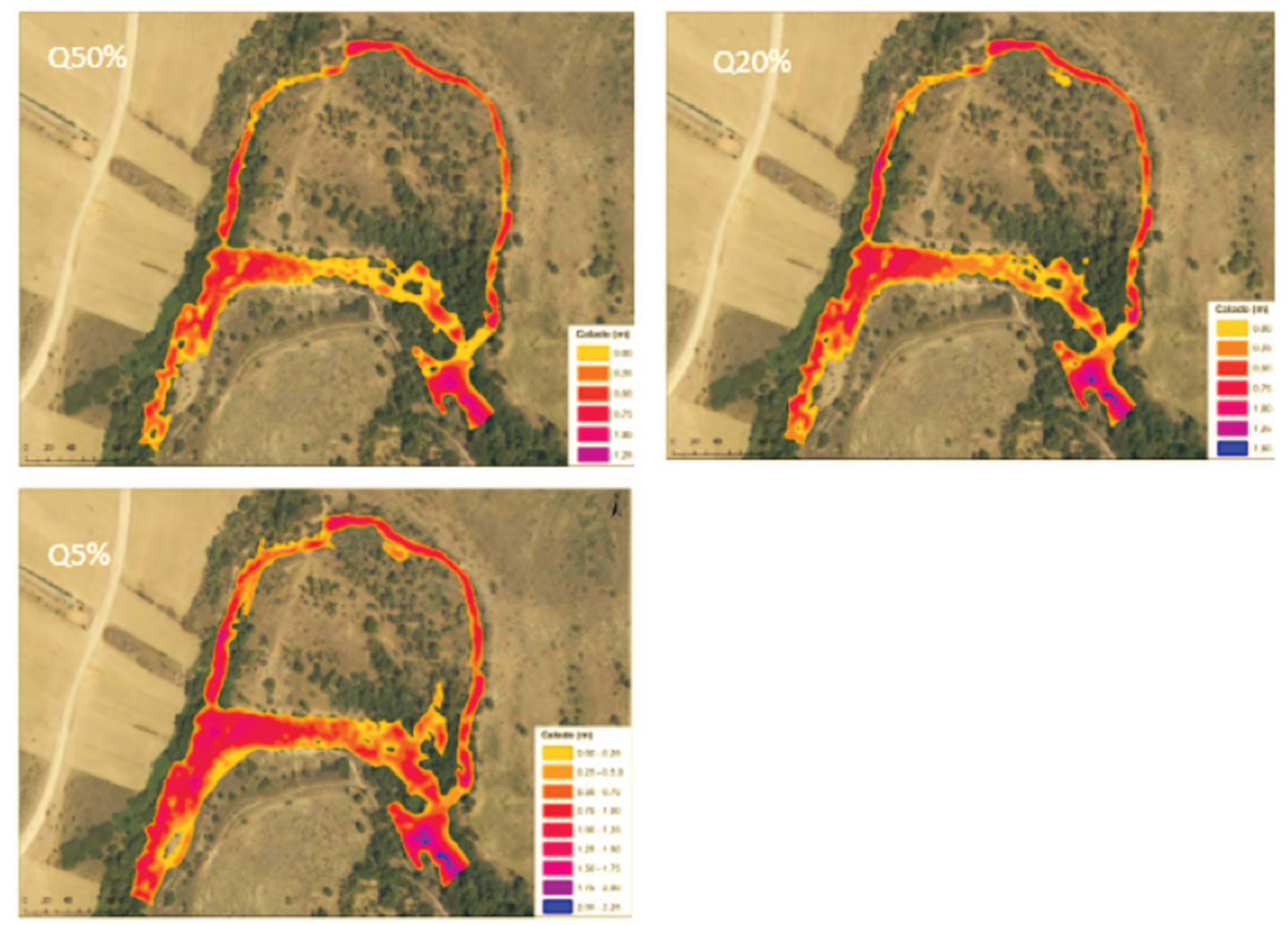

Figura 9. Resultado de los calados alcanzados cuando circulan los caudales Q50\%, Q20\% y Q5\% en el escenario 1.

\subsection{Escenario 2. Reconexión del cauce principal (E1) y activación de cauces secundarios.}

Con las modificaciones propuestas en este escenario no se consigue la activación de cauces secundarios para los caudales Q50\% y Q20\% y estos solamente se activan en avenidas singulares (caudal Q5\%). Por otro lado, los calados obtenidos cuando se activan los cauces secundarios están, mayoritariamente por debajo de los $50 \mathrm{~cm}$ (Figura 10), de modo que se planteó un nuevo escenario que permitiera una reconexión más eficaz de los cauces secundarios. 


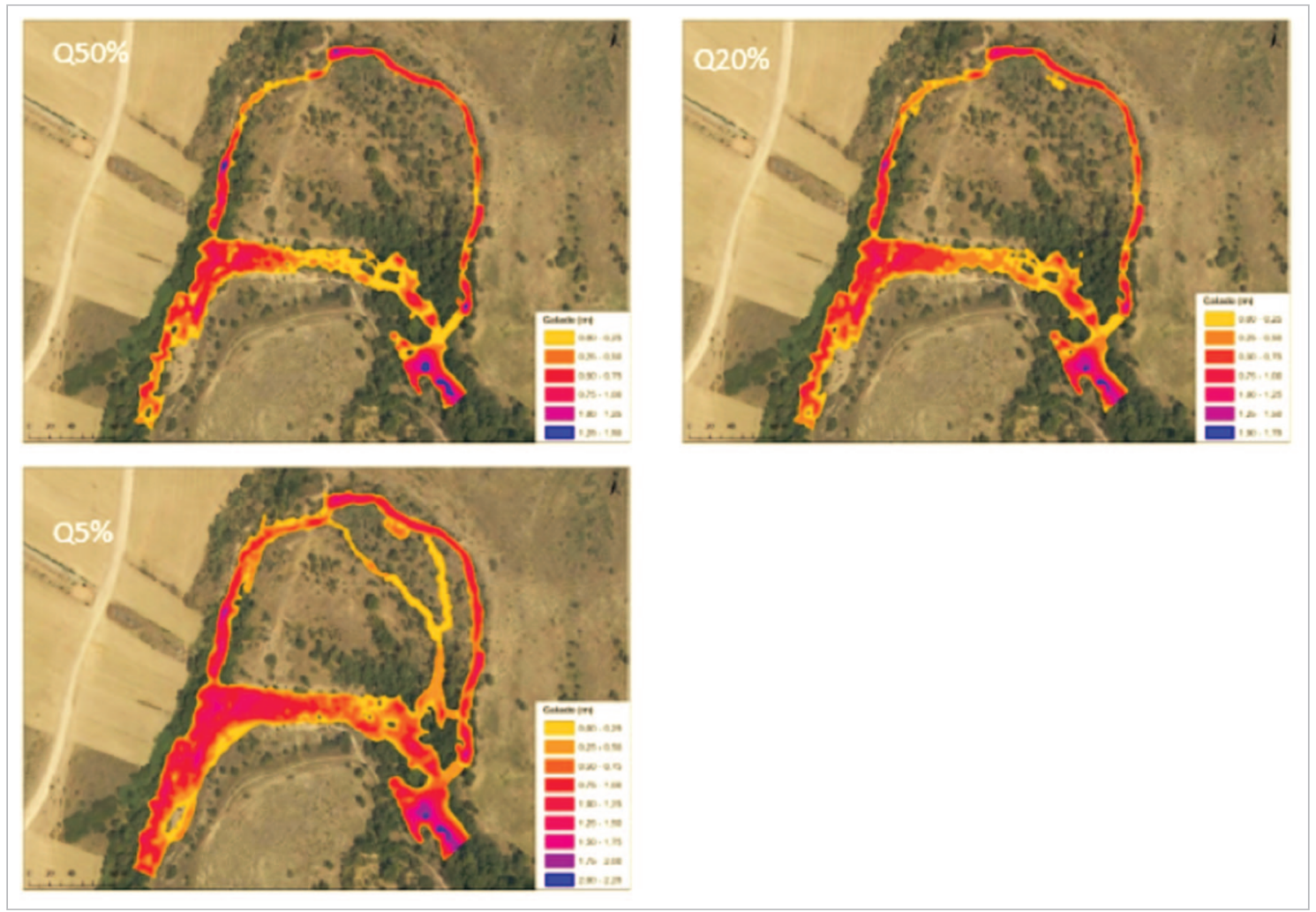

Figura 10. Resultado de los calados alcanzados cuando circulan los caudales Q50\%, Q20\% y Q5\% en el escenario 2 .

3.4. Escenario 3. Reconexión del cauce principal (E1), activación de cauces secundarios (E2) y optimización de la circulación en cauce principal y secundarios.

En el caso del escenario 3, el cauce secundario CS1 se activa para los tres caudales simulados, mientras que el cauce secundario CS2 se activa para Q20\% y Q5\%. Asimismo, se logra que para Q5\%, los calados en el brazo principal y secundarios del meandro presenten, de manera generalizada, valores superiores a los $50 \mathrm{~cm}(\mathrm{Fi}$ gura 11). 


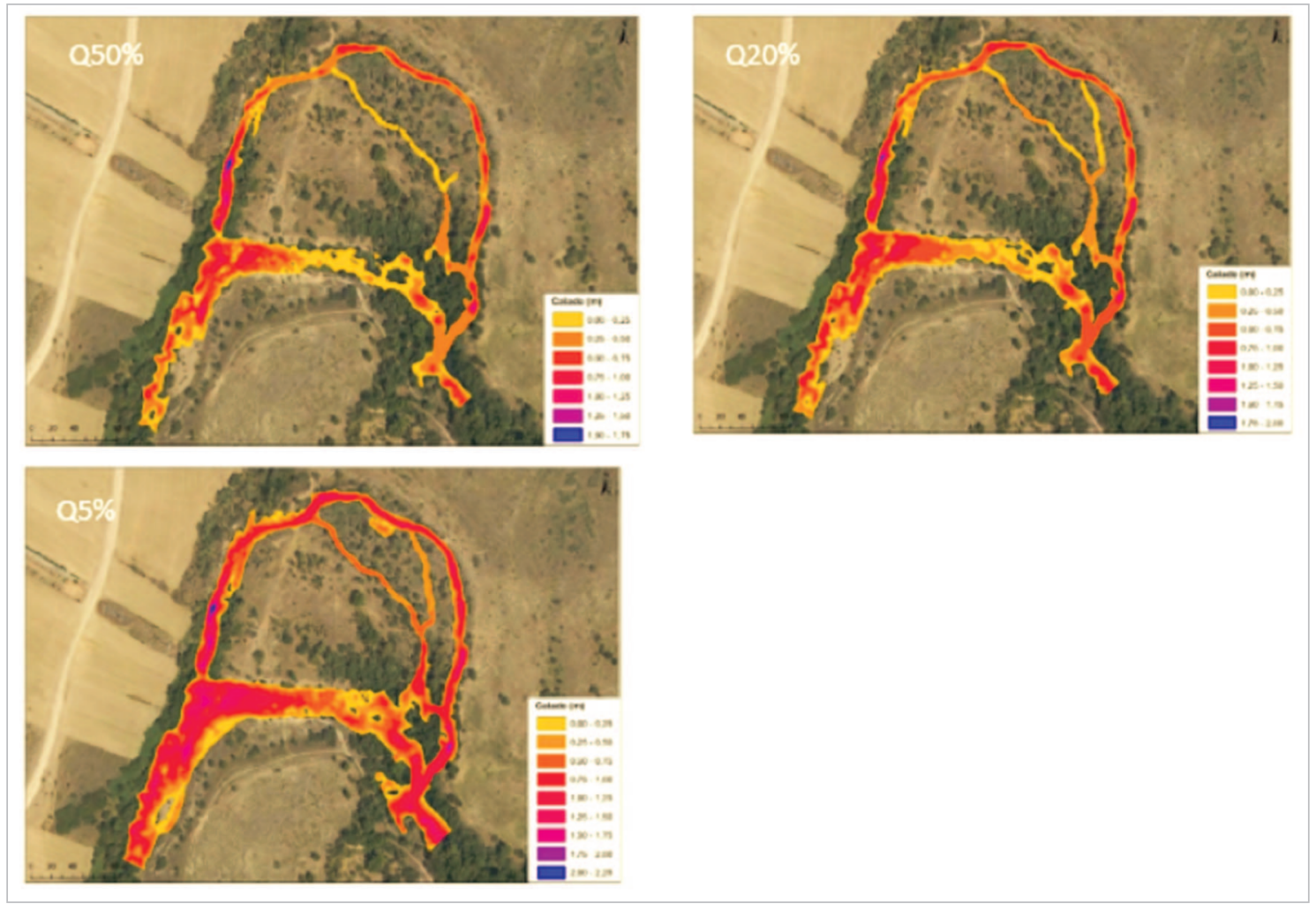

Figura 11. . Resultado de los calados alcanzados cuando circulan los caudales Q50\%, Q20\% y Q5\% en el escenario 3.

\subsection{Indicadores ambientales}

Aunque resultan cualitativamente evidentes las diferencias que cada escenario genera respecto al anterior, se creyó conveniente establecer indicadores objetivos que permitieran cuantificarlas.

Estos indicadores se clasificaron en:

a) Indicadores ambientales

Se escogieron 3 variables trascendentes:

1. La longitud de cauce reconectado, como variable que permite tener una referencia cuantitativa de la rehabilitación de espacio ripario funcional.

2. La superficie mojada, como referencia del lecho de cauce rehabilitado (biotopo hiporréico).

3. El volumen de agua que ocupan los nuevos cauces, como indicador del biotopo acuático incorporado al tramo. 
b) Indicadores de coste

Se consideró el volumen de tierra que había que mover en cada escenario como indicador del coste económico que tendría la actuación.

En la Tabla 4 se presentan los resultados de los indicadores obtenidos para cada escenario y caudal simulado.

Tabla 4. Resultado de los indicadores obtenidos en cada escenario.

\begin{tabular}{|c|c|c|c|c|c|}
\hline & & $\begin{array}{c}\text { Longitud } \\
\text { thalweg }(\mathrm{m})\end{array}$ & $\begin{array}{c}\text { Superficie } \\
\text { mojada }\left(\mathrm{m}^{2}\right)\end{array}$ & $\begin{array}{l}\text { Vol agua } \\
\left(\mathrm{m}^{3}\right)\end{array}$ & $\begin{array}{l}\text { Movimiento } \\
\text { de tierras }\left(\mathrm{m}^{3}\right)\end{array}$ \\
\hline Escenario 0 & $\begin{array}{c}\text { Q5\% } \\
\text { Q20\% } \\
\text { Q50\% }\end{array}$ & $\begin{array}{c}297,3 \\
113,2 \\
0,0\end{array}$ & $\begin{array}{c}3484 \\
495 \\
0\end{array}$ & $\begin{array}{c}1783 \\
125,9 \\
0\end{array}$ & 0 \\
\hline Escenario 1 & $\begin{array}{c}\text { Q5\% } \\
\text { Q20\% } \\
\text { Q50\% }\end{array}$ & $\begin{array}{l}677,5 \\
613,0 \\
613,0\end{array}$ & $\begin{array}{l}7677 \\
4812 \\
4076\end{array}$ & $\begin{array}{l}4804 \\
2390 \\
1875\end{array}$ & 131,2 \\
\hline Escenario 2 & $\begin{array}{c}\text { Q5\% } \\
\text { Q20\% } \\
\text { Q50\% }\end{array}$ & $\begin{array}{c}1012,4 \\
613,0 \\
613,0\end{array}$ & $\begin{array}{l}9650 \\
4777 \\
4123 \\
\end{array}$ & $\begin{array}{l}5435 \\
2366 \\
1923 \\
\end{array}$ & 561,2 \\
\hline Escenario 3 & $\begin{array}{c}\text { Q5\% } \\
\text { Q20\% } \\
\text { Q50\% }\end{array}$ & $\begin{array}{c}1012,4 \\
1007,9 \\
911,6\end{array}$ & $\begin{array}{l}9723 \\
7289 \\
6256\end{array}$ & $\begin{array}{l}6973 \\
3628 \\
2736\end{array}$ & 1765,5 \\
\hline
\end{tabular}

\section{Conclusiones}

Como era de esperar, el escenario 3 es el más favorable desde el punto de vista hidráulico y ambiental. Desde el punto de vista económico, el escenario 3 también es el que requiere un movimiento de tierras mayor, con un volumen de tierras a extraer 3 veces mayor que el del escenario 2 . Sin embargo, ese "coste" implica mejorar sensiblemente (sus valores se multiplican por 1,5) los tres indicadores ambientales, tanto para caudales ordinarios (Q50\%) como para avenidas ordinarias (Q20\%). En consecuencia, se propone el escenario 3 como referente para el proyecto de rehabilitación hidráulica y ambiental del meandro "Los Garridos".

\section{Bibliografía}

Beechie, T. J., Sear, D. A., Olden, J. D., Pess, G. R., Buffington, J. M., Moir, H., Roni, P., Pollock, M. M. (2010). Process-Based Principles for Restoring River Ecosystems. BioScience, 60: 209-222. https://doi.org/10.1525/bio.2010.60.3.7

Bertalan, L., Rodrigo-Comino, J., Surian, N.,_ulc Michalková, M., Kovács, Z., Szabó, S., Szabó, 
G., Hooke, J. (2019). Detailed assessment of spatial and temporal variations in river channel changes and meander evolution as a preliminary work for effective floodplain management. The example of Sajó River, Hungary. Journal of Environmental Management, 248: 1-19. https://doi.org/10.1016/j.jenvman.2019.109277

Bladé, E., Cea, L., Corestein, G., Escolano, E., Puertas, J., Vázquez-Cendón, E., Dolz, J., Coll, A. (2014). Iber: herramienta de simulación numérica del flujo en ríos. Revista Internacional de Métodos Numéricos para Cálculo y Diseño en Ingeniería, 30: 1-10. https://doi.org/10.1016/j.rimni.2012.07.004

Brookes, A. (1987). Restoring the sinuousity of artificially straightened stream channels. Environmental Geology and Water Science 10: 33-41. https://doi.org/10.1007/BF02588003

Brookes, A., Shields, F. D. (1966). River Channel Restoration. Guided Principles for Sustainable Projects.

Bullón, T. (2017). Morfología fluvial del río Jarama entre los siglos XVI y XIX. Datos documentales y cartográficos.

Centro de Estudios hidrográficos. Anuario de aforos 2016-2017. CEDEX.

Centro Ibérico de Restauración Fluvial (CIREF) (2010). ¿Qué es restauración fluvial? Notas Técnicas del CIREF, $n^{\circ} 4$.

De Anta, A., de Castro, M.E., Fompedriña, D. (2018). Depurar para recuperar. Hacia un sector agroganadero sostenible. Congreso Nacional del Medio Ambiente 2018.

García, F., Bernal, A., Carrillo, J.M., López, J., Ochoa, J. (2019). Estrategia para la intervención de recuperación ambiental del valle aluvial del río Segura y sus antiguos meandros a su paso por Murcia. Restaura Ríos 2019 - III Congreso Ibérico de Restauración Fluvial.

González del Tánago, M., García de Jalón, D. (1995). Principios básicos para la restauración de ríos y riberas. Ecología, 9: 34-46.

González del Tánago, M., García de Jalón, D. (2007). Restauración de Ríos. Guía metodológica para la elaboración de Proyectos. Ministerio de Medio Ambiente.

Horvat, Z., Horvat, M., Majer, F., Koch, D. (2020). Hydraulic analysis of a meander on the Danube River using a 2D flow model. Environmental Monitoring and Assessment, 192: 149. https://doi.org/10.1007/s10661-020-8074-z

Kondolf, G. M. (2006). River restoration and meanders. Ecology and Society, 11(2): 42. https://doi.org/10.5751/ES-01795-110242

Magdaleno, F. (2008). Manual de técnicas de restauración fluvial. CEDEX.

Magdaleno, F., Delacámara, G. (2015). Las Medidas Naturales de Retención de Agua: del diseño a la implementación a través de proyectos europeos. Ingeniería civil, 179: 131-138.

Magdaleno, F., Martínez, R. (2006). Aplicaciones de la teledetección láser (LiDAR) en la caracterización y gestión del medio fluvial. Ingeniería Civil, 142: 1-15

Martinez, C., Fernández, J. A. (2008). IAHRIS Índices de Alteración Hidrológica en Ríos. Manual de Referencia Metodológica.

Ministerio de Medio Ambiente y Medio Rural y Marino (2012). Guía Metodológica para el Desarrollo del Sistema Nacional de Cartografía de Zonas Inundables

Ollero, A. (2011). Restauración fluvial: principios, dificultades y propuestas. La perspectiva del CIREF. Restaura Ríos 2011 - I Congreso Ibérico de Restauración Fluvial.

Ollero, A., Ibisate, A., Acín, V., Díaz, E., Granado, D., Horacio, J. (2011). Innovación y libertad fluvial. VII Congreso Ibérico sobre Gestión y Planificación del Agua "Ríos Ibéricos +10 . Mirando al futuro tras 10 años de DMA". 
Rinaldi, M., Johnson, P.A. (1997). Stream meander restoration. JAWRA Journal of the American Water Resources Association, 33: 855-866. https://doi.org/10.1111/j.17521688.1997.tb04110.x

Vizcaíno, P., Magdaleno, F., Seves, A., Merino de Miguel, S., González del Tánago, M., García de Jalón, D. (2003). Los cambios geomorfológicos del río Jarama como base para su restauración. Limnetica, 22: 1-8.

Vogel, R. M. \& Fennessey, N. M. (1995) Flow duration curves. 2: A review of applications in water resources planning. Water Resour. Bull. 31, 1029-1039. https://doi.org/10.1111/j.1752-1688.1995.tb03419.x

Williams, P.B. (2001). River engineering versus river restoration. ASCE Wetlands Engineering \& River Restoration Conference, Reno, American Society of Civil Engineers.

Wohl, E., Angermeier, P. L., Bledsoe, B., Kondolf, G. M., MacDonnell, L., Merritt, D. M., Palmer, M. A., Poff, N. L., Tarboton, D. (2005). River restoration. Water Resources Research, 41, W10301. https://doi.org/10.1029/2005WR003985 
